\title{
MANFREDA PARVA (AGAVACEAE), ESPECIE NUEVA DEL ESTADO DE GUERRERO, MÉXICO
}

\author{
AARÓN RoDRÍGUEZ \\ Universidad de Guadalajara, Instituto de Botánica, \\ Departamento de Botánica y Zoología, \\ Centro Universitario de Ciencias Biológicas y Agropecuarias \\ Apdo. postal 1-139 \\ 45101 Zapopan, Jalisco, México. \\ rca08742@cucba.udg.mx
}

\begin{abstract}
RESUMEN
Como resultado de recientes colectas botánicas en México, se describe a Manfreda parva del estado de Guerrero. Esta especie nueva se caracteriza por sus hojas pequeñas, lineares y falcadas. Pertenece al grupo de $M$. guttata y por la forma de sus hojas es similar a $M$. bulbulifera. Sin embargo, difiere de ésta en el número de hojas, el tamaño de los estambres, el tubo del perianto y los lóbulos del mismo. Se discuten sus afinidades y diferencias morfológicas. Manfreda parva se conoce únicamente de la Sierra de Taxco cercana a la ciudad del mismo nombre.
\end{abstract}

Palabras clave: Agavaceae, Manfreda, México.

\begin{abstract}
As a result of recent botanical collections in Mexico, Manfreda parva is described as a new species from the state of Guerrero. It is characterized by its small, linear and falcate leaves. Manfreda parva belongs to the $M$. guttata group and based on leaf characteristics it is similar to M. bulbulifera. However, M. parva has more leaves, the filaments are shorter, the perianth tube is larger and the perianth lobes are smaller than in M. bulbulifera. So far, the new species is known only from the Sierra de Taxco near the town of the same name.
\end{abstract}

Key words: Agavaceae, Manfreda, Mexico. 


\section{INTRODUCCIÓN}

La familia Agavaceae Endl. tiene una distribución geográfica restringida al continente americano. En México, se reconocen cuando menos 342 especies agrupadas en ocho géneros: Agave L., Beschorneria Kunth, Furcraea Vent., Hesperaloë Engelm., Manfreda Salisb., Polianthes L., Prochnyanthes S. Watson y Yucca L. (García-Mendoza y Galván, 1995). Recientemente, algunos autores incluyen a Hesperoyucca (Engelm.) Baker en la misma familia (Bogler et al., 2006; Good-Avila et al., 2006).

Manfreda se distribuye desde el este y sureste de los Estados Unidos de América (Virginia, Maryland, Indiana, Missouri, Arkansas, Florida y Texas) hasta Honduras y El Salvador (Verhoek-Williams, 1978; Williams, 1998; Rocha et al., 2006). El taxon está formado por más de 30 especies (Piña-Luján, 1985; Espejo y López-Ferrari, 1993; García-Mendoza y Galván, 1995; Rodríguez y Castro-Castro, 2007), de las que 29 se registran de nuestro país. Así, al igual que los demás géneros de agaváceas, Manfreda concentra manifiestamente su diversidad en México (García-Mendoza y Galván, 1995; Williams, 1998; Rodríguez y Castro-Castro, 2007). El número de componentes conocidos de Manfreda ha aumentado a medida que se intensifica la exploración botánica en el país (García-Mendoza et al., 2000; Castillejos-Cruz y Solano, 2007; Hernández-Sandoval et al., 2008). En septiembre del 2007 se colectaron unas plantas pequeñas de Manfreda en las cercanías de Taxco, Guerrero. Después de su examen morfológico se concluyó que se trata de una especie nueva para la cual se propone el siguiente nombre:

Manfreda parva Aaron Rodr., sp. nov. (Figs. 1 y 2).

Herba perennis; cormus 1-4 cm longus, 1-2 cm latus; bulbus oblongus 2-2.5 cm longus, 1-1.5 cm latus; folia (5)11-24, linearia, semisucculenta, plerumque falcata 10-20(25) cm longa, 4-7(9) $\mathrm{mm}$ lata; inflorescentiae parte fertile 15-30 cm longa, laxa, (4)9-15 floribus; quisque flos $3.5 \mathrm{~cm}$ longus; ovario $7 \mathrm{~mm}$ longo, $4 \mathrm{~mm}$ lato; perianthii tubo 1.5-1.8 cm longo, $4.5 \mathrm{~mm}$ diametro, ad apicem ovarii constrictione destituto; segmentis oblongis 7-8 mm longis, 4-5 mm latis, reflexis; capsula 12-17.5 mm longa, 8-13 mm diametro, subglobosa, humero cicatrice manifesta.

Planta herbácea, perenne de 40-60 cm de alto; cormo de 1-4 cm de largo, 1-2 cm de diámetro, con raíces contráctiles carnosas y fibrosas; bulbo de 2-2.5 cm de largo, 1-1.5 cm de diámetro en la base, oblongo, cubierto por las bases de las 


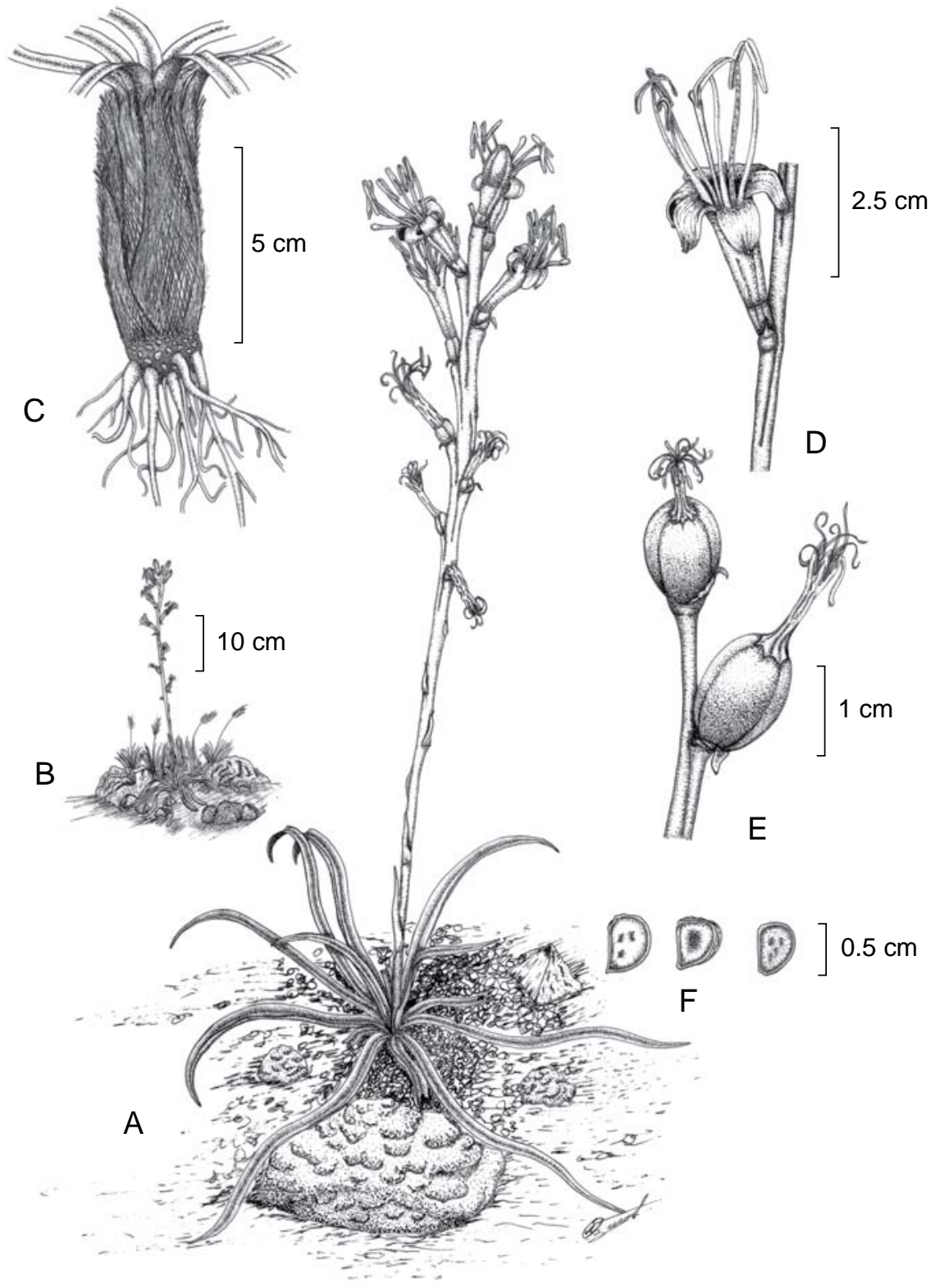

Fig. 1. Manfreda parva. A y B. hábito; C. cormo y bulbo; D. flor; E. frutos; F. semillas. (A. Rodríguez et al. 5211, IBUG). 

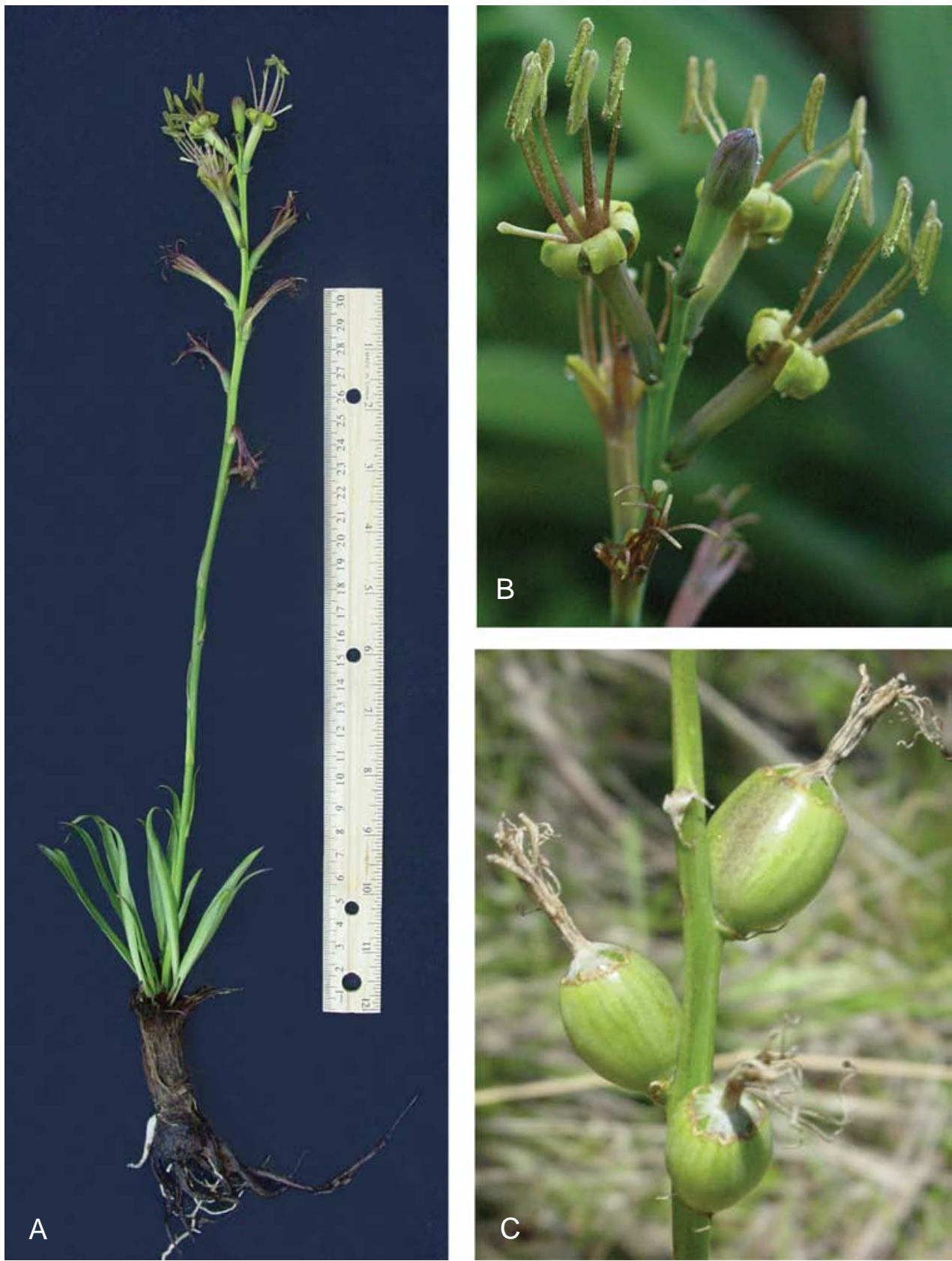

Fig. 2. Manfreda parva. A. hábito; B. flores; C. frutos (A. Rodríguez et al. 5211, IBUG). 
hojas secas, éstas de 6-6.5 cm de largo, membranáceas en la base y con la parte superior fibrosa; hojas del periodo de crecimiento anterior persistentes pero secas; hojas (5)11-24, de 10-20(25) cm de largo, 4-7(9) mm de ancho, extendidas, semisuculentas, lineares, falcadas, verdes, glabras, margen entero, con una banda hialina muy angosta y sólo visible al microscopio, sin papilas, ápice agudo; inflorescencia de 35-60 cm de largo, erecta, porción fértil de 15-30 cm de largo, laxa, con (4)915 flores, eje de color verde en toda su longitud, brácteas 4-7 en la parte estéril, la tercera bráctea basal de (2)4-5 cm de largo, 7-8 $\mathrm{mm}$ de ancho en la base, brácteas florales de 8-9 mm de largo, $6 \mathrm{~mm}$ de ancho, ovadas, apiculadas; flores de $3.5 \mathrm{~cm}$ de largo, difusas, ascendentes, sésiles, verdes con máculas pequeñas, rojizas; tubo del perianto de 1.5-1.8 cm de largo, 4-5 mm de diámetro, sin constricción en el ápice del ovario, recto o ligeramente curvado hacia el exterior, segmentos de 7-8 $\mathrm{mm}$ de largo, 4-5 mm de ancho, oblongos, reflexos, ápice semisuculento, cuculado, con un mechón de tricomas blancos; los filamentos exceden al tubo por $2.5 \mathrm{~cm}$, adnados a la boca del tubo, libres desde la base de los lóbulos, dispuestos en un mismo nivel, erectos en la antesis, verdes con máculas rojizas; anteras de 10-11 mm de largo, 1-1.5 mm de ancho, verdes con máculas rojizas; ovario de $7 \mathrm{~mm}$ de largo y $4 \mathrm{~mm}$ de diámetro, obclaviforme, verde con máculas rojizas; estilo alcanzando hasta la mitad del largo de los estambres, posteriormente se alarga y llegan a la altura de los mismos, estigma trilobado, verde-amarillento; fruto capsular, de $12-17.5 \mathrm{~mm}$ de largo, 8-13 mm de diámetro, subgloboso, triquetro, ápice con cicatriz circular de 4-6 mm de diámetro, con restos secos del perianto, base del estilo persistente en el fruto en forma de un pico de 3-5 mm de largo; semillas de 4.5-5 mm de largo, 3.5 de ancho, plano-cóncavas, negras, brillantes.

Tipo: México, Guerrero: municipio de Taxco, $3 \mathrm{~km}$ al NE de Taxco, a lo largo de la carretera de cuota, justo en El Mirador. Alt. 1845 m. 7.IX.2007, A. Rodríguez et al. 5211 (Holótipo: IBUG, isótipos: ENCB, IEB, MEXU, WIS).

Parátipos: México, Guerrero: municipio de Taxco, 3 km al NE de Taxco, a lo largo de la carretera de cuota, justo en El Mirador. Alt. 1845 m. 27.VII.2008, A. Rodríguez \& L. F. Pérez-Álvarez 5518 (CHAPA, ENCB, FEZA, IBUG, IEB, MEXU, WIS, XAL).

Distribución y hábitat: Manfreda parva crece cerca de Taxco, Guerrero. Habita en laderas rocosas de poca pendiente en bosques de pino y encino perturbado y a una altitud de 1845 m. En el sitio son evidentes Agave horrida Jaco- 
bi, Dodonea viscosa Jacq., Juniperus deppeana Steud. y Mammillaria haageana Pfeiff.

Fenología: florece en julio y fructifica de agosto a septiembre. El epíteto específico se refiere al tamaño pequeño de las plantas.

Las relaciones filogenéticas entre las especies de Manfreda no han sido determinadas. Sin embargo, Verhoek-Williams (1975) propuso grupos de especies con base en características foliares y florales. Manfreda parva es única por sus hojas pequeñas, lineares y falcadas, pero sus características florales nos permiten asociarla con el conjunto de $M$. guttata (Jacobi et Bouché) Rose, donde se encuentran: M. bulbulifera Castillejos \& E. Solano, M. guttata, M. littoralis García-Mend., A. Castañeda et S. Franco, M. pringlei Rose, M. planifolia (S. Watson) Rose y M. rubescens Rose (Verhoek-Williams, 1975; García-Mendoza et al., 2000; Castillejos-Cruz y Solano, 2007). En todas estas especies el ápice del ovario se prolonga hacia el interior de la base del tubo del perianto. En M. guttata, M. littoralis, M. planifolia, M. pringlei y $M$. rubescens las flores se disponen de forma compacta hacia la parte distal de la inflorescencia. En contraste, en M. bulbulifera y M. parva la espiga es laxa y fértil desde la parte media del eje.

Manfreda parva y $M$. bulbulifera tienen hojas lineares pero el número de éstas es mayor en la primera. Otras diferencias entre estas dos especies se resumen en el Cuadro 1.

La presencia de Manfreda parva cerca de la ciudad de Taxco muestra la importancia de las exploraciones botánicas. Martínez-Gordillo et al. (2007) elaboraron un inventario de la flora vascular de la Sierra de Taxco. Sus resultados muestran la presencia de 1794 especies y 16 taxones infra-específicos agrupados en 653 géneros y 159 familias. De acuerdo con los autores, Agavaceae está representada por cinco especies que incluyen $M$. scabra (Ort.) McVaugh y M. pringlei. Aparentemente, $M$. parva pasó desapercibida, a pesar de su cercanía a la ciudad de Taxco.

\section{AGRADECIMIENTOS}

Agradezco a Osvaldo Zuno la elaboración de la figura 1. También deseo expresar mi gratitud a Luis Fernando Pérez Álvarez, Mary Gerritsen y Ron Parsons por su ayuda durante el trabajo de campo. Por último, doy gracias a Roberto González Tamayo por su ayuda con la diagnosis en latín. 
Rodríguez: Manfreda parva, especie nueva de Guerrero, México

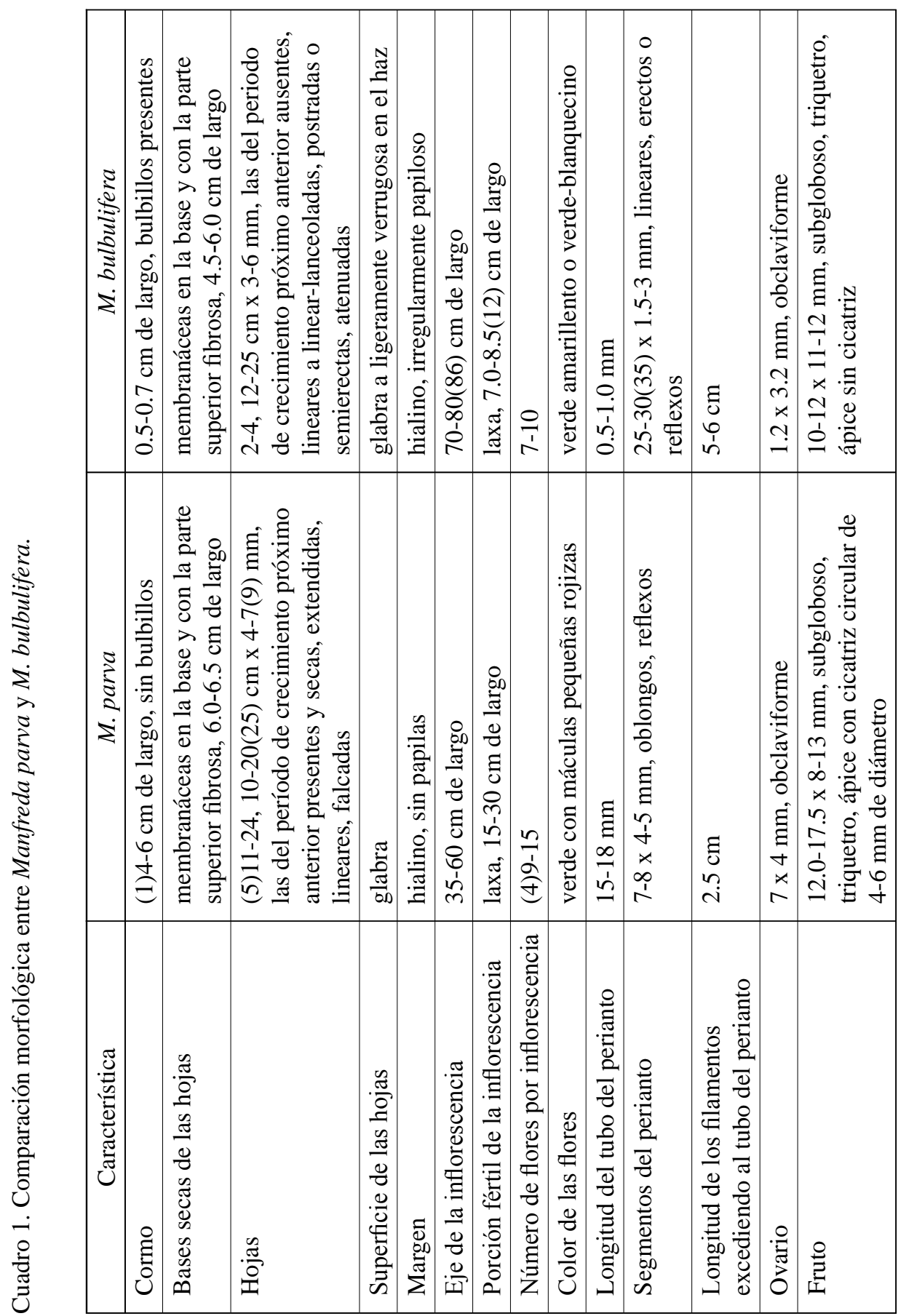




\section{LITERATURA CITADA}

Bogler, D. J., J. C. Pires y J. Francisco-Ortega. 2006. Phylogeny of Agavaceae based on $n d h \mathrm{~F}, r b c \mathrm{~L}$, and ITS sequences: implications of molecular data for classification. Aliso 22: 313-328.

Castillejos-Cruz, C. y E. Solano. 2007. Manfreda bulbulifera (Agavaceae), especie nueva de México. Acta Bot. Mex. 82: 67-73.

Espejo, A. y A. R. López-Ferrari. 1993. Las monocotiledóneas mexicanas. Una sinopsis florística. Lista de referencia. Parte 1. Consejo Nacional de la Flora de México. Universidad Autónoma Metropolitana. México D.F. pp. 33-36.

García-Mendoza, A. y R. Galván. 1995. Riqueza de las familias Agavaceae y Nolinaceae en México. Bol. Soc. Bot. Méx. 56: 7-24.

García-Mendoza, A., A. Castañeda-Rojas e I. S. Franco-Martínez. 2000. Manfreda littoralis (Agavaceae), nueva especie de Guerrero y Oaxaca, México. Acta Bot. Mex. 50: 39-45.

Good-Avila, S. V., V. Souza, B. S. Gaut y L. E. Eguiarte. 2006. Timing and rate of speciation in Agave (Agavaceae). Proc. Natl. Acad. Sci. U.S.A. 103: 9124-9129.

Hernández-Sandoval, L., R. Orellana y G. Carnevali. 2008. Two new species of Manfreda Salisb. (Agavaceae) from the Yucatán Peninsula, Mexico. Jour. Torr. Bot. Soc. 135: 168-177.

Martínez-Gordillo, M., R. Cruz-Durán y C. A. Ruiz-Jiménez. 2007. Flora vascular de la Sierra de Taxco. In: Luna, I., J. J. Morrone y D. Espinosa (eds.). Diversidad de la Faja Volcánica Transmexicana. Universidad Nacional Autónoma de México. México, D.F. pp. 149-171.

Piña-Luján, I. 1985. Consideraciones sobre el género Manfreda I. Cact. Suc. Mex. 30(2): 27-32.

Rocha, M., S. Good-Avila, F. Molina-Freaner, H. T. Arita, M. Castillo, A. García-Mendoza, A. Silva-Montellano, B. S. Gaut, V. Souza y L. E. Eguiarte. 2006. Pollination biology and adaptive radiation of Agavaceae, with special emphasis on the genus Agave. Aliso 22: 329-344.

Rodríguez, A. y A. Castro-Castro. 2007. Potencial ornamental de los “amoles” (Manfreda, Agavaceae) en México. Ibugana 15(1): 3-11.

Verhoek-Williams, S. 1975. A study of the tribe Poliantheae (including Manfreda) and revision of Manfreda and Prochnyanthes (Agavaceae). Ph.D. Thesis. Cornell University. Ithaca, Nueva York. 405 pp.

Verhoek-Williams, S. 1978. Huaco and amole: a survey of the uses of Manfreda and Prochnyanthes. Econ. Bot. 32: 124-130.

Williams, S. 1998. Agavaceae. In: Kubitzki, K. (ed.). The families and genera of vascular plants III: flowering plants. Monocotiledons, Lilianae (except Orchidaceae). Springer. Berlin. pp. 60-70. 